\title{
Integral mean estimates for the polar derivative of polynomials whose zeros are within a circle
}

Ahmad Zireh*, Elahe Khojastehnezhad and S Reza Musawi

"Correspondence:

azireh@shahroodut.ac.ir

Department of Mathematics,

Shahrood University of Technology,

Shahrood, Iran

\section{Abstract}

For a polynomial $p(z)$ of degree $n$, having all zeros in $|z| \leq k$, where $k \leq 1$, Dewan et al. (Southeast Asian Bull. Math. 34:69-77, 2010) proved that for every $\alpha \in \mathbb{C}$ with $|\alpha| \geq k$ and for each $r>0$,

$$
n(|\alpha|-k)\left\{\int_{0}^{2 \pi}\left|p\left(e^{i \theta}\right)\right|^{r} d \theta\right\}^{\frac{1}{r}} \leq\left\{\int_{0}^{2 \pi}\left|1+k e^{i \theta}\right|^{r} d \theta\right\}^{\frac{1}{r}} \max _{|z|=1}\left|D_{\alpha} p(z)\right| .
$$

In this paper we improve and extend the above inequality. Our result generalizes certain well-known polynomial inequalities.

MSC: Primary 30A10; secondary 30C10; 30D15

Keywords: polar derivative; polynomial; inequality; maximum modulus; restricted zeros

\section{Introduction and statement of results}

Let $p(z)$ be a polynomial of degree $n$. Then according to Bernstein's inequality [1] on the derivative of a polynomial, we have

$$
\max _{|z|=1}\left|p^{\prime}(z)\right| \leq n \max _{|z|=1}|p(z)| \text {. }
$$

This result is best possible and equality holds for a polynomial that has all zeros at the origin.

If we restrict to the class of polynomials which have all zeros in $|z| \leq 1$, then it has been proved by Turán [2] that

$$
\max _{|z|=1}\left|p^{\prime}(z)\right| \geq \frac{n}{2} \max _{|z|=1}|p(z)| .
$$

The inequality (1.2) is sharp and equality holds for a polynomial that has all zeros on $|z|=1$. As an extension to (1.2), Malik [3] proved that if $p(z)$ has all zeros in $|z| \leq k$, where $k \leq 1$, then

$$
\max _{|z|=1}\left|p^{\prime}(z)\right| \geq \frac{n}{1+k} \max _{|z|=1}|p(z)| .
$$

This result is best possible and equality holds for $p(z)=(z-k)^{n}$. 
On the other hand, Malik [4] obtained a generalization of (1.2) in the sense that the righthand side of (1.2) is replaced by a factor involving the integral mean of $p(z)$ on $|z|=1$. In fact he proved that if $p(z)$ has all its zeros in $|z| \leq 1$, then for each $r>0$,

$$
n\left[\int_{0}^{2 \pi}\left|p\left(e^{i \theta}\right)\right|^{r} d \theta\right]^{\frac{1}{r}} \leq\left[\int_{0}^{2 \pi}\left|1+e^{i \theta}\right|^{r} d \theta\right]^{\frac{1}{r}} \max _{|z|=1}\left|p^{\prime}(z)\right| .
$$

As an extension of (1.4), Aziz [5] proved that if $p(z)$ has all its zeros in $|z| \leq k \leq 1$, then for each $r>0$,

$$
n\left[\int_{0}^{2 \pi}\left|p\left(e^{i \theta}\right)\right|^{r} d \theta\right]^{\frac{1}{r}} \leq\left[\int_{0}^{2 \pi}\left|1+k e^{i \theta}\right|^{r} d \theta\right]^{\frac{1}{r}} \max _{|z|=1}\left|p^{\prime}(z)\right|
$$

As a generalization of (1.5), Aziz and Shah [6] proved that if $p(z)=a_{n} z^{n}+\sum_{v=\mu}^{n} a_{n-v} z^{n-v}$, $1 \leq \mu \leq n$, is a polynomial of degree $n$, having all its zeros in $|z| \leq k \leq 1$, then for each $r>0$,

$$
n\left[\int_{0}^{2 \pi}\left|p\left(e^{i \theta}\right)\right|^{r} d \theta\right]^{\frac{1}{r}} \leq\left[\int_{0}^{2 \pi}\left|1+k^{\mu} e^{i \theta}\right|^{r} d \theta\right]^{\frac{1}{r}} \max _{|z|=1}\left|p^{\prime}(z)\right| .
$$

Let $D_{\alpha} p(z)$ denote the polar derivative of the polynomial $p(z)$ of degree $n$ with respect to $\alpha \in \mathbb{C}$. Then $D_{\alpha} p(z)=n p(z)+(\alpha-z) p^{\prime}(z)$. The polynomial $D_{\alpha} p(z)$ is of degree at most $n-1$ and it generalizes the ordinary derivative in the sense that

$$
\lim _{\alpha \rightarrow \infty}\left[\frac{D_{\alpha} p(z)}{\alpha}\right]=p^{\prime}(z)
$$

Shah [7] extended (1.2) to the polar derivative of $p(z)$ and proved that if all zeros of the polynomial $p(z)$ lie in $|z| \leq 1$, then for every $\alpha$ with $|\alpha| \geq 1$, we have

$$
\max _{|z|=1}\left|D_{\alpha} p(z)\right| \geq \frac{n}{2}(|\alpha|-1) \max _{|z|=1}|p(z)|
$$

This result is best possible and equality holds for $p(z)=(z-1)^{n}$ with $\alpha \geq 1$.

Aziz and Rather [8] extended the inequality (1.3) to the polar derivative of a polynomial. In fact, they proved that if all zeros of $p(z)$ lie in $|z| \leq k, k \leq 1$, then for every $\alpha$ with $|\alpha| \geq k$, we get

$$
\max _{|z|=1}\left|D_{\alpha} p(z)\right| \geq \frac{n}{1+k}(|\alpha|-k) \max _{|z|=1}|p(z)|
$$

This result is best possible and equality holds for $p(z)=(z-k)^{n}$ with $\alpha \geq k$.

Recently Dewan et al. [9] generalized the inequalities (1.5) and (1.8). They proved that if $p(z)$ has all its zeros in $|z| \leq k \leq 1$, then for every $\alpha \in \mathbb{C}$ with $|\alpha| \geq k$ and for each $r>0$,

$$
n(|\alpha|-k)\left\{\int_{0}^{2 \pi}\left|p\left(e^{i \theta}\right)\right|^{r} d \theta\right\}^{\frac{1}{r}} \leq\left\{\int_{0}^{2 \pi}\left|1+k e^{i \theta}\right|^{r} d \theta\right\}^{\frac{1}{r}} \max _{|z|=1}\left|D_{\alpha} p(z)\right| .
$$

In the limiting case, when $r \rightarrow \infty$, the above inequality is sharp and equality holds for the polynomial $p(z)=(z-k)^{n}$ with $\alpha \geq k$. 
The following result which we prove is a generalization as well as a refinement of inequalities (1.9) and (1.8). In a precise set up, we have the following.

Theorem 1.1 If $p(z)=a_{n} z^{n}+\sum_{v=\mu}^{n} a_{n-v} z^{n-v}, 1 \leq \mu \leq n$, is a polynomial of degree $n$, having all its zeros in $|z| \leq k \leq 1$ and $m=\min _{|z|=k}|p(z)|$, then for $\lambda, \alpha \in \mathbb{C}$ with $|\lambda| \leq 1,|\alpha| \geq s_{\mu}$ and $r>0, d>1, q>1$, with $\frac{1}{d}+\frac{1}{q}=1$, we have

$$
\begin{aligned}
& n\left(|\alpha|-s_{\mu}\right)\left[\int_{0}^{2 \pi}\left|p\left(e^{i \theta}\right)+\lambda m\right|^{r} d \theta\right]^{\frac{1}{r}} \\
& \quad \leq\left[\int_{0}^{2 \pi}\left|1+s_{\mu} e^{i \theta}\right|^{d r} d \theta\right]^{\frac{1}{d r}}\left[\int_{0}^{2 \pi}\left|D_{\alpha} p\left(e^{i \theta}\right)\right|^{q r} d \theta\right]^{\frac{1}{q r}},
\end{aligned}
$$

where $s_{\mu}=\frac{n\left|a_{n}\right| k^{2 \mu}+\mu\left|a_{n-\mu}\right| k^{\mu-1}}{n\left|a_{n}\right| k^{\mu-1}+\mu\left|a_{n-\mu}\right|}$. In the limiting case, when $r \rightarrow \infty$, the above inequality is sharp and equality holds for the polynomial $p(z)=(z-k)^{n}$ with $\alpha \geq k$.

Letting $q \rightarrow \infty$ (so that $d \rightarrow 1$ ) in (1.10), we have the following.

Corollary 1.2 If $p(z)=a_{n} z^{n}+\sum_{v=\mu}^{n} a_{n-v} z^{n-v}, 1 \leq \mu \leq n$, is a polynomial of degree $n$, having all its zeros in $|z| \leq k \leq 1$ and $m=\min _{|z|=k}|p(z)|$, then for $\lambda, \alpha \in \mathbb{C}$ with $|\lambda| \leq 1,|\alpha| \geq s_{\mu}$ and $r>0$,

$$
n\left(|\alpha|-s_{\mu}\right)\left[\int_{0}^{2 \pi}\left|p\left(e^{i \theta}\right)+\lambda m\right|^{r} d \theta\right]^{\frac{1}{r}} \leq\left[\int_{0}^{2 \pi}\left|1+s_{\mu} e^{i \theta}\right|^{r} d \theta\right]^{\frac{1}{r}} \max _{|z|=1}\left|D_{\alpha} p(z)\right|,
$$

where $s_{\mu}$ is defined as in Theorem 1.1.

Remark 1.3 Since by Lemma 2.3, $s_{\mu} \leq k$, the inequality (1.11) provides a refinement and generalization of the inequality (1.9).

If we divide both sides of the inequality (1.11) by $|\alpha|$ and make $|\alpha| \rightarrow \infty$, we obtain the following refinement and generalization of the inequality (1.6).

Corollary 1.4 If $p(z)=a_{n} z^{n}+\sum_{\nu=\mu}^{n} a_{n-v} z^{n-v}, 1 \leq \mu \leq n$, is a polynomial of degree $n$, having all its zeros in $|z| \leq k \leq 1$ and $m=\min _{|z|=k}|p(z)|$, then for every $\lambda \in \mathbb{C}$ with $|\lambda| \leq 1$ and $r>0$,

$$
n\left[\int_{0}^{2 \pi}\left|p\left(e^{i \theta}\right)+\lambda m\right|^{r} d \theta\right]^{\frac{1}{r}} \leq\left[\int_{0}^{2 \pi}\left|1+s_{\mu} e^{i \theta}\right|^{r} d \theta\right]^{\frac{1}{r}} \max _{|z|=1}\left|p^{\prime}(z)\right|,
$$

where $s_{\mu}$ is defined as in Theorem 1.1.

Letting $r \rightarrow \infty$ in (1.10) and choosing the argument of $\lambda$ suitably with $|\lambda|=1$, we have the following result.

Corollary 1.5 If $p(z)=a_{n} z^{n}+\sum_{v=\mu}^{n} a_{n-v} z^{n-v}, 1 \leq \mu \leq n$, is a polynomial of degree $n$, having all its zeros in $|z| \leq k \leq 1$, then for every $\alpha \in \mathbb{C}$ with $|\alpha| \geq s_{\mu}$,

$$
\frac{n\left(|\alpha|-s_{\mu}\right)}{1+s_{\mu}}\left[\max _{|z|=1}|p(z)|+\min _{|z|=k}|p(z)|\right] \leq \max _{|z|=1}\left|D_{\alpha} p(z)\right|
$$

where $s_{\mu}$ is defined as in Theorem 1.1. 


\section{Lemmas}

For the proof of the theorem, the following lemmas are needed. The first lemma is due to Laguerre [10].

Lemma 2.1 If all the zeros of an nth degree polynomial $p(z)$ lie in a circular region $C$ and $w$ is any zero of $D_{\alpha} p(z)$, then at most one of the points $w$ and $\alpha$ may lie outside $C$.

Lemma 2.2 If $p(z)=a_{n} z^{n}+\sum_{v=\mu}^{n} a_{n-\nu} z^{n-\nu} ; 1 \leq \mu \leq n$, is a polynomial of degree $n$ having all its zeros in $|z| \leq k \leq 1$ and $q(z)=z^{n} \overline{p\left(\frac{1}{\bar{z}}\right)}$, then on $|z|=1$

$$
\left|q^{\prime}(z)\right| \leq s_{\mu}\left|p^{\prime}(z)\right|
$$

and

$$
\frac{\mu}{n}\left|\frac{a_{n-\mu}}{a_{n}}\right| \leq k^{\mu}
$$

where $s_{\mu}=\frac{n\left|a_{n}\right| k^{2 \mu}+\mu\left|a_{n-\mu}\right| k^{\mu-1}}{n\left|a_{n}\right| k^{\mu-1}+\mu\left|a_{n-\mu}\right|}$.

The above lemma is due to Aziz and Rather [8].

Lemma 2.3 If $p(z)=a_{n} z^{n}+\sum_{v=\mu}^{n} a_{n-\nu} z^{n-v}, 1 \leq \mu \leq n$, has all its zeros in $|z| \leq k, k \leq 1$, then

$$
s_{\mu} \leq k^{\mu},
$$

where $s_{\mu}$ is same as above.

Proof By using Lemma 2.2, we have

$$
\frac{\mu}{n}\left|\frac{a_{n-\mu}}{a_{n}}\right| \leq k^{\mu}
$$

or

$$
\mu\left|a_{n-\mu}\right| \leq n\left|a_{n}\right| k^{\mu}
$$

or equivalently,

$$
\mu\left|a_{n-\mu}\right|-n\left|a_{n}\right| k^{\mu} \leq 0 .
$$

Since $k \leq 1$ and $\mu \geq 1$, the above inequality implies

$$
\left(k^{\mu-1}-k^{\mu}\right)\left(\mu\left|a_{n-\mu}\right|-n\left|a_{n}\right| k^{\mu}\right) \leq 0,
$$

that is,

$$
\mu\left|a_{n-\mu}\right| k^{\mu-1}-n\left|a_{n}\right| k^{\mu} k^{\mu-1}-\mu\left|a_{n-\mu}\right| k^{\mu}+n\left|a_{n}\right| k^{2 \mu} \leq 0,
$$


which is equivalent to

$$
\mu\left|a_{n-\mu}\right| k^{\mu-1}+n\left|a_{n}\right| k^{2 \mu} \leq\left(n\left|a_{n}\right| k^{\mu-1}+\mu\left|a_{n-\mu}\right|\right) k^{\mu},
$$

which implies

$$
s_{\mu}=\frac{n\left|a_{n}\right| k^{2 \mu}+\mu\left|a_{n-\mu}\right| k^{\mu-1}}{n\left|a_{n}\right| k^{\mu-1}+\mu\left|a_{n-\mu}\right|} \leq k^{\mu} .
$$

Lemma 2.4 If $p(z)=a_{n} z^{n}+\sum_{v=\mu}^{n} a_{n-\nu} z^{n-\nu}, 1 \leq \mu \leq n$, is a polynomial of degree $n$, having all zeros in the closed disk $|z| \leq k, k \leq 1$, then for every real or complex number $\alpha$ with $|\alpha| \geq s_{\mu}$ and $|z|=1$, we have that

$$
\left|D_{\alpha} p(z)\right| \geq\left(|\alpha|-s_{\mu}\right)\left|p^{\prime}(z)\right|
$$

where $s_{\mu}$ is same as above.

Proof Let $q(z)=z^{n} \overline{p(1 / \bar{z})}$, then $\left|q^{\prime}(z)\right|=\left|n p(z)-z p^{\prime}(z)\right|$ on $|z|=1$. Thus on $|z|=1$, we get

$$
\begin{aligned}
\left|D_{\alpha} p(z)\right| & =\left|n p(z)+(\alpha-z) p^{\prime}(z)\right|=\left|\alpha p^{\prime}(z)+n p(z)-z p^{\prime}(z)\right| \\
& \geq\left|\alpha p^{\prime}(z)\right|-\left|n p(z)-z p^{\prime}(z)\right|,
\end{aligned}
$$

which implies

$$
\left|D_{\alpha} p(z)\right| \geq|\alpha|\left|p^{\prime}(z)\right|-\left|q^{\prime}(z)\right| \text {. }
$$

By combining (2.1) and (2.6), we obtain

$$
\left|D_{\alpha} p(z)\right| \geq\left(|\alpha|-s_{\mu}\right)\left|p^{\prime}(z)\right|
$$

\section{Proof of the theorem}

Proof of Theorem 1.1 If $k=0$, then $p(z)$ has all its zeros at the origin, therefore $p(z)=a_{n} z^{n}$. In this case $m=0, s_{\mu}=0$ and $D_{\alpha} P(z)=n \alpha a_{n} z^{n-1}$, therefore on the left-hand side of (1.10), we have

$$
n\left(|\alpha|-s_{\mu}\right)\left[\int_{0}^{2 \pi}\left|p\left(e^{i \theta}\right)+\lambda m\right|^{r} d \theta\right]^{\frac{1}{r}}=n|\alpha|\left|a_{n}\right|(2 \pi)^{\frac{1}{r}}
$$

and on the right-hand side of (1.10) we have

$$
\begin{aligned}
& {\left[\int_{0}^{2 \pi}\left|1+s_{\mu} e^{i \theta}\right|^{d r} d \theta\right]^{\frac{1}{d r}}\left[\int_{0}^{2 \pi}\left|D_{\alpha} p\left(e^{i \theta}\right)\right|^{q r} d \theta\right]^{\frac{1}{q r}}} \\
& \quad=(2 \pi)^{\frac{1}{d r}} n|\alpha|\left|a_{n}\right|(2 \pi)^{\frac{1}{q r}}=n|\alpha|\left|a_{n}\right|(2 \pi)^{\frac{1}{r}\left(\frac{1}{d}+\frac{1}{q}\right)}=n|\alpha|\left|a_{n}\right|(2 \pi)^{\frac{1}{r}} .
\end{aligned}
$$


Therefore, in the case $k=0$, Theorem 1.1 is true. So, we suppose that $k>0$, which implies $s_{\mu}>0$. Let $q(z)=z^{n} \overline{p\left(\frac{1}{\bar{z}}\right)}$, then on $|z|=1$, we have

$$
\left|p^{\prime}(z)\right|=\left|n q(z)-z q^{\prime}(z)\right|
$$

Let $m=\min _{|z|=k}|p(z)|$. Now $m \leq|p(z)|$ for $|z|=k$, therefore, if $\lambda$ is any real or complex number such that $|\lambda|<1$, then

$$
|\lambda m|<|p(z)| \text { for }|z|=k
$$

Since all the zeros of $p(z)$ lie in $|z| \leq k$, it follows by Rouche's theorem that all the zeros of

$$
F(z)=p(z)-\lambda m
$$

also lie in $|z| \leq k$. If $G(z)=z^{n} \overline{F\left(\frac{1}{\bar{z}}\right)}=q(z)+\bar{\lambda} m z^{n}$, then by applying Lemma 2.2 to $F(z)$, we have

$$
\left|G^{\prime}(z)\right| \leq s_{\mu}\left|F^{\prime}(z)\right| \text { for }|z|=1
$$

that is,

$$
\left|q^{\prime}(z)+\bar{\lambda} n m z^{n-1}\right| \leq s_{\mu}\left|p^{\prime}(z)\right|
$$

Now using (3.1) in the above inequality, we get

$$
\left|q^{\prime}(z)+\bar{\lambda} n m z^{n-1}\right| \leq s_{\mu}\left|n q(z)-z q^{\prime}(z)\right|
$$

Since $p(z)$ has all its zeros in $|z| \leq k \leq 1$, by the Gauss-Lucas theorem all the zeros of $p^{\prime}(z)$ also lie in $|z| \leq k \leq 1$. This implies that the polynomial

$$
z^{n-1} \overline{p^{\prime}\left(\frac{1}{\bar{z}}\right)}=n q(z)-z q^{\prime}(z)
$$

has all its zeros in $|z| \geq \frac{1}{k} \geq 1$.

Therefore, it follows from (3.3) and (3.4) that the function

$$
w(z)=\frac{z\left(q^{\prime}(z)+\bar{\lambda} n m z^{n-1}\right)}{s_{\mu}\left(n q(z)-z q^{\prime}(z)\right)}
$$

is analytic for $|z| \leq 1$, and $|w(z)| \leq 1$ for $|z| \leq 1$. Furthermore, $w(0)=0$. Thus the function

$$
1+s_{\mu} w(z)
$$

is subordinate to the function

$$
1+s_{\mu} z
$$

for $|z| \leq 1$. 
Hence by a well-known property of subordination [11], we have for each $r>0$ and $0 \leq$ $\theta \leq 2 \pi$,

$$
\int_{0}^{2 \pi}\left|1+s_{\mu} w\left(e^{i \theta}\right)\right|^{r} d \theta \leq \int_{0}^{2 \pi}\left|1+s_{\mu} e^{i \theta}\right|^{r} d \theta .
$$

Also from (3.5), we have

$$
1+s_{\mu} w(z)=\frac{n\left(q(z)+\bar{\lambda} m z^{n}\right)}{n q(z)-z q^{\prime}(z)} .
$$

Therefore

$$
n\left|q(z)+\bar{\lambda} m z^{n}\right|=\left|1+s_{\mu} w(z)\right|\left|n q(z)-z q^{\prime}(z)\right| .
$$

Since $\left|q(z)+\bar{\lambda} m z^{n}\right|=|p(z)+\lambda m|$ for $|z|=1$, we get from (3.7) and (3.1)

$$
n|p(z)+\lambda m|=\left|1+s_{\mu} w(z)\right|\left|p^{\prime}(z)\right| \text { for }|z|=1 .
$$

From (2.5) and (3.8), we have

$$
n\left(|\alpha|-s_{\mu}\right)|p(z)+\lambda m| \leq\left|1+s_{\mu} w(z)\right|\left|D_{\alpha} p(z)\right| \quad \text { for }|z|=1 .
$$

By combining (3.6) and (3.9), for each $r>0$, we get

$$
\begin{gathered}
\left(n\left(|\alpha|-s_{\mu}\right)\right)^{r} \int_{0}^{2 \pi}\left|p\left(e^{i \theta}\right)+\lambda m\right|^{r} d \theta \\
\leq \int_{0}^{2 \pi}\left|1+s_{\mu} e^{i \theta}\right|^{r}\left|D_{\alpha} p\left(e^{i \theta}\right)\right|^{r} d \theta .
\end{gathered}
$$

Now applying Holder's inequality for $d>1, q>1$, with $\frac{1}{d}+\frac{1}{q}=1$ to (3.10), we get

$$
\begin{aligned}
& n\left(|\alpha|-s_{\mu}\right)\left[\int_{0}^{2 \pi}\left|p\left(e^{i \theta}\right)+\lambda m\right|^{r} d \theta\right]^{\frac{1}{r}} \\
& \quad \leq\left[\int_{0}^{2 \pi}\left|1+s_{\mu} e^{i \theta}\right|^{d r} d \theta\right]^{\frac{1}{d r}}\left[\int_{0}^{2 \pi}\left|D_{\alpha} p\left(e^{i \theta}\right)\right|^{q r} d \theta\right]^{\frac{1}{q r}},
\end{aligned}
$$

which is the desired result.

\section{Competing interests}

The authors declare that they have no competing interests.

\section{Authors' contributions}

The authors declare that they have no competing interests. All authors read and approved the final manuscript.

\section{Acknowledgements}

The authors are grateful to the referees for the careful reading of the paper and for the helpful suggestions and comments.

Received: 6 July 2012 Accepted: 20 May 2013 Published: 3 July 2013 


\section{References}

1. Bernstein, S: Leons sur les propriétés extrémales et la meilleure approximation des fonctions analytiques d'une variable réelle. Gauthier-Villars, Paris (1926)

2. Turán, P: Über die ableitung von Polynomen. Compos. Math. 7, 89-95 (1939)

3. Malik, MA: On the derivative of a polynomial. J. Lond. Math. Soc. 1, 57-60 (1969)

4. Malik, MA: An integral mean estimate for polynomials. Proc. Am. Math. Soc. 91, 281-284 (1984)

5. Aziz, A: Integral mean estimate for polynomials with restricted zeros. J. Approx. Theory 55, $232-239$ (1988)

6. Aziz, A, Shah, WM: An integral mean estimate for polynomials. Indian J. Pure Appl. Math. 28, 1413-1419 (1997)

7. Shah, WM: A generalization of a theorem of Paul Turán. J. Ramanujan Math. Soc. 1, 67-72 (1996)

8. Aziz, A, Rather, NA: A refinement of a theorem of Paul Turán, concerning polynomials. Math. Inequal. Appl. 1, 231-238 (1998)

9. Dewan, KK, Singh, N, Mir, A, Bhat, A: Some inequalities for the polar derivative of a polynomial. Southeast Asian Bull. Math. 34, 69-77 (2010)

10. Laguerre, E: Oeuvres de Laquerre, vol. 1, 2nd edn, pp. 48-66. Chelsea, New York (1898)

11. Hille, E: Analytic Function Theory, II. Ginn and Company, New York, Toronto (1962)

doi:10.1186/1029-242X-2013-307

Cite this article as: Zireh et al.: Integral mean estimates for the polar derivative of polynomials whose zeros are within a circle. Journal of Inequalities and Applications 2013 2013:307.

\section{Submit your manuscript to a SpringerOpen ${ }^{\mathcal{O}}$ journal and benefit from:}

- Convenient online submission

- Rigorous peer review

Immediate publication on acceptance

- Open access: articles freely available online

- High visibility within the field

- Retaining the copyright to your article 